\title{
TECNOLOGIAS SOCIAIS PARA A PROMOÇÃO DA SAÚDE NA PRIMEIRA INFÂNCIA EM MUNICÍPIOS PAULISTAS
}

\begin{abstract}
Alfredo Almeida Pina-Oliveira
Enfermeiro, Doutorando e Mestre em Ciências da Saúde pela Escola de Enfermagem da Universidade de São Paulo (EEUSP). Coordenador dos grupos educativos do Centro de Promoção da Saúde do Hospital das Clínicas da Faculdade de Medicina da Universidade de São Paulo (CPS-HCFMUSP). Mestre adjunto pleno da Faculdade de Campo Limpo Paulista (FACCAMP). E-mail: aapo@ig.com.br.
\end{abstract}

\section{Anna Maria Chiesa}

Enfermeira, Livre-Docente, Professora Associada do Departamento de Enfermagem em Saúde Coletiva da EEUSP. Consultora Técnica da Fundação Maria Cecília Souto Vidigal

(FMCSV). E-mail: amchiesa@usp.br.

\section{RESUMO}

Promover a saúde na primeira infância consiste em uma estratégia inteligente e criativa que fortalece o desenvolvimento social e econômico. $\mathrm{O}$ presente artigo objetivou apresentar a construção de tecnologias sociais para o cuidado integral e integrado. Trata-se de um relato da experiência da Fundação Maria Cecília Souto Vidigal na composição de oito intervenções-chave intersetoriais e interprofissionais em municípios do interior paulista. Discute-se o referencial empregado para a reformulação das políticas e programas de saúde, educação e assistência social com ênfase nos três primeiros anos. Conclui-se que as tecnologias sociais destinadas à promoção do desenvolvimento infantil incorporam inovações no cuidado das gestantes e das crianças de zero a três anos sem desconsiderar suas famílias e suas comunidades.

PALAVRAS-CHAVE: Cuidado da criança, desenvolvimento infantil, promoção da saúde, difusão de inovações, organizações não governamentais.

\section{SOCIAL TECHNOLOGIES TOWARDS EARLY CHILD DEVELOPMENT PROMOTION IN MUNICIPALITIES AT MUNICIPALITIES OF SÃO PAULO, BRAZIL}

\section{SUMMARY}

Promoting healthy early childhood depicts an intelligent and creative strategy in order to improve social and economic development. This paper aims to present social technologies building for integral and integrated care. It is a Third Sector Foundation's experience report about elaboration of intersectorial and interprofessional eight key interventions in municipalities at inner-state of Sao Paulo, Brazil. We have argued about a theoretical model adopted for Health, Education and Welfare policies and programs reform related to the first three years old. We have concluded that social technologies focused on early child development incorporated innovative care destined to pregnants, newborns and toddlers regardless their families and communities. 
KEYWORDS: Child care, child development, health promotion, diffusion of innovations, non-governmental organizations.

\section{TECNOLOGIAS SOCIAIS PARA A PROMOÇÃO DA SAÚDE NA PRIMEIRA INFÂNCIA EM MUNICÍPIOS PAULISTAS}

\section{INTRODUÇÃO}

O Ministério da Ciência e da Tecnologia (MCT) lança a Política Nacional de Ciência, Tecnologia e Inovação em Saúde (PNCTIS), que tem como um dos seus objetivos (BRASIL, 2008, p. 5):

\footnotetext{
"Contribuir para que o desenvolvimento nacional se faça de modo sustentável, e com apoio na produção de conhecimentos técnicos e científicos ajustados às necessidades econômicas, sociais, culturais e políticas do País. (...)”.
}

Nesse mesmo documento, a delimitação do campo da pesquisa em saúde fundamenta-se em "conhecimentos, tecnologias e inovações de cuja aplicação resulte em melhorias na saúde da população" (BRASIL, 2008, p. 5) e deve ser entendida como um "bem da sociedade a ser preservado e desenvolvido por ela, sendo garantido pelo Estado os meios necessários para tal fim" (BRASIL, 2008, p. 6).

Novaes (2006) discute sobre as tecnologias de processo na atenção à saúde que abarcam desde procedimentos com componentes técnicos mais estruturados até formas de cuidado, de educação e de gestão pautados a priori em evidências científicas, sem desconsiderar as contribuições de outros conhecimentos.

As inovações tecnológicas na área de saúde podem ser exemplificadas por medicamentos, equipamentos, procedimentos técnicos, sistemas de apoio, processos educativos, protocolos e programas de saúde (LORENZETTI et al., 2012).

Contudo, baseada em revisão da literatura sobre a produção e a avaliação de tecnologias nos sistemas de saúde, Novaes (2006, p. 137) amplia essa noção:

\footnotetext{
"Muitas das tecnologias de processo atualmente incorporadas à atenção à saúde passaram a ser objeto de pesquisa e avaliação em sistemas e serviços de saúde. Assim, por meio do fortalecimento da base científica da atenção à saúde e da produção de evidências [científicas], procura-se contribuir para a transformação da atenção à saúde".
}

Koerich et al. (2006) referem que o desafio da área de saúde e, em especial, da enfermagem consiste em incorporar e ou adequar as tecnologias aos aspectos éticos e às demandas contemporâneas da sociedade que visa a extrapolação da dimensão tecnológica frente a intersubjetividade do cuidar.

Entende-se como tecnologias sociais "técnicas, materiais e procedimentos metodológicos testados, validados e com impacto social comprovado, criados a partir de necessidades sociais, com o fim de solucionar um problema social" (INSTITUTO DE TECNOLOGIA SOCIAL, 2014).

A necessidade de produzir mudanças no trabalho exige a valorização dos sujeitos na construção social da saúde, pois os profissionais de saúde "modificam seus modos tecnológicos de construir o cuidado, intervindo nas formas de uso de suas ferramentas 
conhecimento e equipamentos" a fim de reorientar os serviços (MERHY, FRANCO, 2008).

As relações intersubjetivas e o agir cotidiano representam o núcleo do trabalho em saúde que permite o "trabalho vivo em ato". O envolvimento dos trabalhadores e dos usuários dos serviços de saúde na construção do cuidado individual e coletivo deve incorporar inovações tecnológicas que não se limita à dimensão técnica e procedimental (MERHY, FRANCO, 2008).

Os profissionais de saúde devem considerar a complementaridade entre as tecnologias duras, equipamentos e recursos materiais; leve-duras, campos do conhecimento e protocolos; e leves, comunicação e relações intersubjetivas, para a produção do cuidado que deve ser compreendida como um processo que ocorre em diferentes graus de mudança (MERHY, FRANCO, 2008).

No campo da promoção do desenvolvimento infantil (PDI), o acúmulo de evidências científicas embasa estratégias inteligentes, criativas, participativas e equitativas a fim de contribuir para a promoção da saúde, da justiça social, do desenvolvimento social e econômico (CENTER, 2014; CYPEL, 2011; MARMOT, ATKINSON \& BELL, 2010; SHONKOFF, 2012; YOUNG, 2010).

À luz da importância da PDI, o presente artigo objetivou apresentar a construção de tecnologias sociais para o cuidado integral e integrado direcionado a gestantes e a crianças de zero a três anos em municípios paulistas partícipes em projetos de intervenção local (PIL) da Fundação Maria Cecília Souto Vidigal (FMCSV).

\section{MATERIAIS E MÉTODO}

Trata-se de um estudo de caso (YIN, 2010) sobre a construção de tecnologias sociais para a construção de oito intervenções-chave (IC) dos PIL da FMCSV (2011) compostos por conteúdos inovadores e temas relevantes para diferentes atores sociais relevantes (profissionais, familiares e cuidadores) envolvidos direta ou indiretamente no cuidado de crianças de zero a três anos, suas famílias e suas comunidades.

Os avanços da Neurociência, da Biologia Molecular e da Epigenética, por exemplo, os exames de neuroimagem, aprofundam a compreensão sobre os efeitos das experiências iniciais no corpo humano; a importância de cuidados que protegem das adversidades, uma vez que estas interferem na citoarquitetura cerebral, nas funções regulatórias do metabolismo e nos sistemas imunológico e cardiovascular; os efeitos destas "interferências" podem persistir e causar prejuízos físicos e psicológicos na vida adulta (CDC, 2010; SHONKOFF, 2012).

Portanto, adotou-se a fundamentação teórico-metodológica (Figura 1) desenvolvida pelo Center on Developing Child, University of Harvard (2010) e traduzida pela equipe técnica da FMCSV (2011) a fim de conferir um caráter inovador da PDI. 


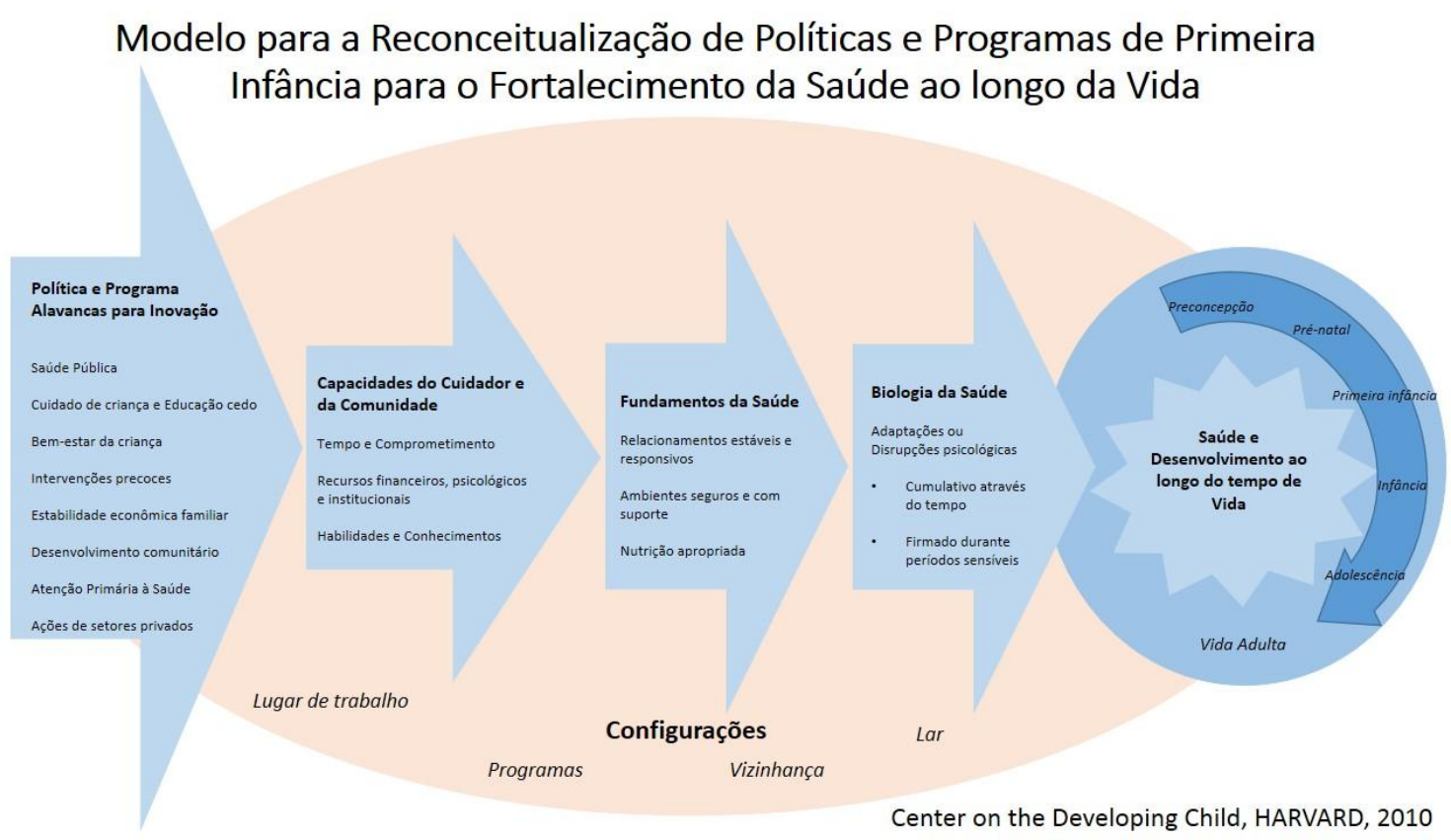

\section{Figura 1 - Referencial teórico-metodológico para políticas e programas com foco na primeira infância. Center on Developing Child, Harvard, 2010.}

Adotou-se o modelo acima para caracterizar as tecnologias sociais da FMCSV representadas pelos conteúdos inovadores das oito IC analisados por Oliveira et al (2013) e que compreendem as inovações tecnológicas como "novos projetos, programas, produtos, mudanças organizacionais, reformas políticas e intervenções sistemáticas (PATTON, 2011, p. 1)" relacionadas ao alcance da sustentabilidade antes de se tornar obsoleta.

A inovação provoca surpresa e está vinculada à novidade, nem sempre à invenção. $O$ desenvolvimento social e econômico prima por estratégias inovadoras, pois como afirma Arbix (2010, p. 171):

\footnotetext{
"O conhecimento sempre foi essencial para o desenvolvimento das sociedades. Isso não significa, no entanto, que seu lugar, características e dinâmica sejam exatamente os mesmos ao longo da história da humanidade. Há não mais do que trinta anos o conhecimento começou a sofrer drásticas mudanças, tanto em sua composição como em sua geração, combinação e difusão. Atualmente, o conhecimento transformou-se no sistema nervoso central do desenvolvimento social e político em todos os cantos do mundo".
}

A compreensão do contexto torna-se fundamental para a construção de práticas suportadas por inovações tecnológicas e evidências científicas em prol da promoção da saúde e da qualidade de vida da população (LAROUCHE, POTVIN, 2013; GREENHALGH, WIERINGA, 2011).

Bojer (2013, p. 6) afirma que "a inovação é dar vida a ideia. A criatividade pode ser individual, a inovação requer colaboração". Para compreender a aplicação desse conceito em ações programáticas, a autora realiza a distinção entre a inovação social e a inovação técnica (Tabela 1) a seguir:

Tabela 1. Características da inovação social e da inovação técnica.

\begin{tabular}{|l|l|}
\hline Inovação Social & Inovação Técnica \\
\hline Resposta à complexidade social & Resposta à complexidade técnica \\
\hline
\end{tabular}




\begin{tabular}{|l|ll|}
\hline $\begin{array}{l}\text { Dependente das escolhas das } \\
\text { pessoas }\end{array}$ & $\begin{array}{l}\text { Pode “funcionar" independente da } \\
\text { adoção do mercado }\end{array}$ \\
\hline Altamente dependente do contexto & Depende menos do contexto & \\
\hline $\begin{array}{l}\text { Envolve de } \\
\text { comportamento e modelo mental }\end{array}$ & $\begin{array}{l}\text { Não requer a aprovação de } \\
\text { tecnologias existentes }\end{array}$ \\
\hline $\begin{array}{l}\text { Deve ser testada em um sistema } \\
\text { vivo }\end{array}$ & $\begin{array}{l}\text { Pode ser testada em um laboratório } \\
\text { fechado }\end{array}$ \\
\hline
\end{tabular}

Fonte: Bojer, 2013.

Nota-se que a complementaridade entre as inovações técnicas e sociais pode potencializar o processo de difusão de inovações como algo não linear, incerto, emergente, dinâmico, adaptativo e coevolutivo permite ao avaliador abordar as interfaces de um fenômeno cujas variáveis não estão sob seu controle e ou são bastante imprevisíveis (PATTON, 2011).

A construção de tecnologias sociais para o cuidado integral e integrado da FMCSV considerou os contextos locais e a as características da população contribuem para a construção de boas práticas a fim de traduzir as evidências científicas em alternativas promotoras da saúde, em soluções para a inclusão social e em formas de melhoria da qualidade de vida (FMCSV, 2011, 2013).

\section{RESULTADOS E DISCUSSÃO}

A Fundação Maria Cecília Souto Vidigal (FMCSV) desenvolve o programa "Primeiríssima Infância", antigo Programa de Desenvolvimento Infantil, a fim de valorizar cuidados qualificados desde a concepção até os primeiros três anos de vida a partir de uma abordagem interprofissional e intersetorial com as famílias e as comunidades (FMCSV, 2011).

Esse programa contrapõe-se às ações programáticas dedicadas à PDI que atuam de forma fragmentada, ora na saúde, ora na educação, ora nos demais setores (Cypel, 2011). Para tanto, foram criados os Projetos de Intervenção Local (PIL) da FMCSV, anteriormente denominados Projetos Comunitários (FMCSV, 2011). O acervo digital e demais registros desse programa estão disponíveis em: http://www.fmcsv.org.br/pt-br/Paginas/default.aspx.

Com foco no investimento na PDI, a missão da FMCSV está pautada em "desenvolver a criança para desenvolver a sociedade" e a visão dessa instituição está fundamentada em "gerar e disseminar conhecimento para o desenvolvimento integral da criança" (FMCSV, 2011).

Dessa forma, a FMCSV atua em três focos estratégicos e complementares, a articulação de parcerias, a gestão do conhecimento e o aprimoramento das práticas (CYPEL, 2011; FMCSV, 2013).

Os PIL enfatizam o cuidado que contempla as necessidades essenciais da criança em seus aspectos emocionais, a capacitação de profissionais para incorporar tais conceitos inovadores, a defesa pelas ações de promoção da saúde sustentadas por políticas públicas municipais e pelo fortalecimento das competências familiares (CHIESA et al., 2009; CYPEL, 2011; FMCSV, 2011; SHONKOFF, 2012). 
Veríssimo e Chiesa (2009) defendem que os profissionais de diferentes áreas devem incorporar temas relacionados ao amor, à brincadeira e à segurança no cuidado infantil que exerce uma função biológica protetora, mas que, sobretudo valoriza os aspectos emocionais, sociais, culturais e ambientais da própria criança e de sua família.

Os municípios do interior paulista que aderiram aos PIL apresentavam critérios específicos que contribuiriam para fortalecer a primeira infância, desejo político das lideranças comunitárias e potencial para o cuidado integral e integrado no atendimento às gestantes, às crianças de zero a três anos, suas famílias e suas comunidades (FMCSV, 2011).

Tal parceria implicou no compromisso entre prefeitos, secretários dos setores saúde, educação e assistência social, membros da comunidade local e representantes das IES para a consolidação dos PIL (FMCSV, 2011).

Para operacionalizar os PIL, cada município partícipe compôs um núcleo de governança local constituído por um Comitê Estratégico formado por prefeito, autoridades do governo municipal e lideranças sociais e por um Comitê Técnico constituído por profissionais das secretarias de saúde, educação, assistência social, organizações não governamentais e representantes de faculdades ou Universidades locais (FMCSV, 2013).

Definiu-se também o papel de secretário executivo que enfatiza a mediação para a implementação dos PIL e a formação de redes parceiras para a consolidação da PDI alicerçadas em tecnologias sociais (FMCSV, 2011, 2013). Sendo assim, o programa "Primeiríssima infância" poderia avançar na elaboração de alternativas viáveis para o enfrentamento de problemas sociais (MACIEL, FERNANDES, 2011) e para a sustentabilidade programática (RODRIGUES, BARBIERI, 2008).

Os serviços locais de saúde, educação e assistência social foram avaliados com base no Early Development Instrument, antes e após a adesão aos PIL, a fim de evidenciar os progressos, as estabilidades e os retrocessos dos indicadores na constituição dessa rede intersetorial em prol da primeira infância. Essa fase foi denominada Marco Zero (FMCSV, 2009, 2011, 2013).

Após o Marco Zero, os consultores técnicos da FMCSV realizaram ações educativas com os atores sociais relevantes dos PIL, sendo denominadas intervenções-chave (IC), quais sejam (FMCSV, 2011) e analisados com maior profundidade por Oliveira et al. (2013):

- IC1. Pré-natal ampliado

- IC2. Grupos de famílias grávidas

- IC3. Parto humanizado

- IC4. Puerpério e aleitamento materno

- IC5. Puericultura ampliada (zero a três anos)

- IC6. Educação infantil em creches

- IC7. Espaços lúdicos

- IC8. Grupos de famílias com crianças de zero a três anos de idade

Essas oito IC reiteram o conceito de tecnologia social e contribuem, segundo Cypel (2011, p. 9), para "a organização dos alicerces que favorecerão o indivíduo, no futuro, a lidar com as complexas situações que a vida venha a lhe apresentar" e ilustram as tecnologias leves para a promoção do cuidado integral e integrado da primeira infância na rede de atenção às gestantes, às crianças de zero a três anos, às famílias e aos demais cuidadores nos 
diferentes setores dos municípios paulistas e proporcionam conteúdos inovadores para a abordagem da primeira infância.

Nesse sentido, a valorização do cuidado integral (baseado em aspectos biológicos, afetivos e socioambientais) e integrado (intersetorial e interprofissional) pode contribuir para compor estratégias inovadoras para a reorientação das práticas nos serviços e fortalecer as famílias e os diferentes atores sociais da comunidade em prol da primeira infância saudável (CHIESA et al., 2009; CYPEL, 2011; VERÍSSIMO, CHIESA, 2009).

As oportunidades para a PDI ocorrem por meio dos relacionamentos estáveis, responsivos, lúdicos e ricos em experiências sensoriais. $\mathrm{O}$ fortalecimento das competências para $\mathrm{o}$ educar, brincar e cuidar da criança nesta fase da vida propicia benefícios permanentes para a aprendizagem, para a socialização, para os comportamentos preventivos e para a saúde física e mental (VERÍSSIMO, CHIESA, 2009; YOUNG, 2010).

Para que a reaplicação em escala fosse atingida, as IC foram divididas em dois momentos. O primeiro foi composto por oficinas de formação com profissionais dos setores saúde, educação e assistência social, bem como outros profissionais envolvidos com a PDI no município (FMCSV, 2011; WHO, 2011).

O segundo foi dividido em três a cinco supervisões periódicas, bimestrais ou trimestrais, a fim de verificar a aplicabilidade e a reedição dos conteúdos inovadores das oito IC com as equipes nos serviços de origem (FMCSV, 2009, 2011, 2013).

As oficinas de formação foram estruturadas com base em metodologias ativas e participativas para a incorporação dos conteúdos inovadores (CHIESA et al., 2009). Não obstante, os participantes comprometiam-se com a realização de reedições em seus locais de origem, IES, serviços locais etc, e o fortalecimento das redes de cuidado integral e integrado (FMCSV, 2011, 2013).

As supervisões periódicas dos consultores das IC da FMCSV prestariam apoio técnico para a valorização dos pontos fortes e para a superação de nós críticos identificados nos projetos de reedição com potencialidade de reaplicação em escala em diversos contextos (FMCSV, 2013; WHO, 2011).

Os contextos das reedições foram as IES, as redes de serviços locais com foco nas gestantes e nas crianças de zero a três anos, sem desconsiderar as interfaces com a família e com a comunidade, tais como os serviços de planejamento familiar, o pré-natal, as maternidades, as unidades de saúde, os centros de convivência social, as creches, os conselhos tutelares, entre outros loci de produção de cuidados (FMCSV, 2011, 2013).

Para promover o aprendizado contínuo posterior às oficinas e às supervisões das IC, a FMCSV realiza eventos científicos anuais e oferece acesso livre e gratuito a livros, enciclopédias, manuais técnicos, vídeos, apresentações dos eventos científicos e radar da primeira infância para os participantes dos PIL e o público em geral (FMCSV, 2013).

A parceria com as IES dos municípios partícipes permite "garantir a continuidade da disseminação dos conteúdos do projeto, mesmo após o período de financiamento da FMCSV (2011, p. 7)", demonstrando o papel estratégico para a garantia da sustentabilidade da PDI por meio da formação acadêmica.

A participação dos representantes das IES - docentes, discentes, coordenadores, técnicoadministrativos e diretores - pode catalisar processos de mudança no ensino, na pesquisa e na extensão (FMCSV, 2011, 2013). Sendo assim, avaliar esse processo pode contribuir para a reorientação das práticas acadêmicas com foco na formação profissional, crítica, reflexiva e cidadã. 
Portanto, existe a necessidade de associar a formação crítica e humanista de profissionais das equipes de saúde da família ao processo introdutório de novas tecnologias de cuidado que enfatizam o diálogo, a co-responsabilização e a autonomia de familiares e cuidadores de crianças de zero a seis anos em ações intersetoriais e interprofissionais (CHIESA et. al, 2009).

\section{CONSIDERAÇÕES FINAIS}

A incorporação das evidências científicas constitui elemento-chave para a tomada de decisão, a elaboração de leis e o desenvolvimento de ações estratégicas em diferentes setores e segmentos sociais a fim de investir em áreas prioritárias para a melhoria das condições de vida da população, nesse caso, a primeira infância.

O referencial teórico e metodológico desenvolvido pelo CDC de Harvard e traduzido pela Fundação Maria Cecília Souto Vidigal (FMCSV) demonstrou alinhamento com o paradigma da Promoção da Saúde com foco na concepção, gestação e primeira infância, sem desconsiderar o contexto de vida, de trabalho e de relacionamentos das famílias e das comunidades.

A experiência exitosa da FMCSV incentivou a utilização e a construção de tecnologias sociais direcionadas ao cuidado integral e integrado com ênfase nos três primeiros anos de vida a fim de promover um processo de mudança efetivo, contextualizado e construtivo para lidar com a complexidade da promoção do desenvolvimento infantil.

$\mathrm{O}$ envolvimento de diferentes atores sociais relevantes nos municípios partícipes com foco no fortalecimento dos recursos e parcerias e no enfrentamento dos nós críticos contribuiu para a consolidação dos projetos de intervenção local e a incorporação dos conteúdos inovadores das oito intervenções-chave.

Conclui-se que o emprego de conteúdos inovadores, que mesclam elementos da inovação técnica e social, associados a projetos intersetoriais e interprofissionais podem potencializar os recursos das redes de atenção à saúde, à educação e à assistência social nos primeiros anos que influenciam sobremaneira os ciclos vitais subsequentes.

\section{AGRADECIMENTOS}

À Fundação de Amparo à Pesquisa do Estado de São Paulo pelo financiamento para a realização da pesquisa intitulada "A universidade e a transferência de tecnologias de desenvolvimento infantil em municípios paulistas" (processo FAPESP n 2010/09263-6) e à Fundação Maria Cecília Souto Vidigal pela oportunidade fortalecer o cuidado integral e integrado da Primeira Infância nos municípios paulistas partícipes do programa "Primeiríssima Infância".

\section{REFERÊNCIAS BIBLIOGRÁFICAS}

ARBIX, G. Estratégias de inovação para o desenvolvimento. Tempo Social - Revista de sociologia da USP, v. 22, n. 2, p. 167-185, 2010. Disponível em: http://www.scielo.br/pdf/ts/v22n2/v22n2a09. Acesso em: 16 ago. 2014.

BRASIL. Ministério da Saúde, Secretaria de Ciência, Tecnologia e Insumos Estratégicos, Departamento de Ciência e Tecnologia. Política nacional de ciência, tecnologia e inovação em saúde. 2. ed. Brasília: Editora do Ministério da Saúde, 2008. 
BOJER, M. Processos colaborativos para inovação social: experiências e aprendizados. In: Anais do III Simpósio Internacional de Desenvolvimento da Primeira Infância da Fundação Maria Cecília Souto Vidigal; 2013 out. São Paulo [Internet]. São Paulo: Fundação Maria Cecília Souto Vidigal; 2013. Disponível em: http://www.fmcsv.org.br/ptbr/acervo-digital/Paginas/Mille-Bojer---Processos-colaborativos-parainova\%C3\%A7\%C3\%A3o-social-experi\%C3\%AAncias-e-aprendizados.aspx. Acesso em 16 ago. 2014.

CENTER ON THE DEVELOPING CHILD. The foundations of lifelong health are built in early childhood. Cambridge, MA: Harvard University; 2010. Disponível em: http://developingchild.harvard.edu/resources/reports_and_working_papers/foundations-oflifelong-health/. Acesso em: 16 ago. 2014.

CHIESA, A.M. et al. A construção de tecnologias de atenção em saúde com base na promoção da saúde. Rev. Esc. Enferm. USP [online], v. 43, n. 2, p. 1352-1357, 2009. Disponível em: http://www.scielo.br/pdf/reeusp/v43nspe2/a36v43s2.pdf. Acesso em: 16 ago. 2014.

CYPEL, S. (Org.). Fundamentos do desenvolvimento infantil: da gestação aos 3 anos. São Paulo: Fundação Maria Cecília Souto Vidigal, 2011.

DIDONET, V. (Org.). Plano Nacional pela Primeira Infância. Brasília: Rede Nacional pela Primeira Infância, 2010. Disponível em: http://primeirainfancia.org.br/wpcontent/uploads/PPNI-resumido.pdf. Acesso em: 16 ago. 2014.

FRENK, J. et al. Health professionals for a new century: transforming education to strengthen health systems in an interdependent world. The Lancet, v. 376, n. 9756, p. 1923-1958, 2010. Disponível em: http://dash.harvard.edu/bitstream/handle/1/4626403/Ed_HealthProfCommisionp5_40.PDF ?sequence=1. Acesso em 16 ago. 2014.

FUNDAÇÃO MARIA CECÍLIA SOUTO VIDIGAL. Projetos de Intervenção Local: Estratégias para qualificar a atenção à Primeira Infância. São Paulo, 2011.

Programa Primeiríssima Infância: estratégias para qualificar a atenção à criança pequena. São Paulo: Fundação Maria Cecília Souto Vidigal; 2013.

GREENHALGH, T.; WIERINGA, S. Is it time to drop the 'knowledge translation' metaphor? A critical literature review. J R Soc Med, v. 104, n. 12, p. 501-509, 2011. DOI: 10.1258/jrsm.2011.110285.

INSTITUTO DE TECNOLOGIA SOCIAL. Reflexões sobre a construção do conceito de tecnologia social. In: DE PAULO, A. et al. Tecnologia social: uma estratégia para o desenvolvimento. Rio de Janeiro: Fundação Banco do Brasil, 2004.

KOERICH M. S. et al. Tecnologias de cuidado em saúde e enfermagem e suas perspectivas filosóficas. Texto Contexto Enferm, v. 15, p. 178-185, 2006. Número Especial. Disponível em: http://www.scielo.br/pdf/tce/v15nspe/v15nspea22. Acesso em: 16 ago. 2014.

LAROUCHE, A.; POTVIN, L. Stimulating innovative research in health promotion. Global Health Promotion, v. 20, n. 2, p. 64-69, 2013. DOI: 10.1177/1757975913490428.

LORENZETTI, J et al. Tecnologia, inovação tecnológica e saúde: uma reflexão necessária. Texto Contexto Enferm, v. 21, n. 2, p. 432-439, 2012. Disponível em: http://www.scielo.br/pdf/tce/v21n2/a23v21n2.pdf. Acesso em: 16 ago. 2014. 
MACIEL, A. L. S.; FERNANDES, R. M. C. Tecnologias sociais: interface com as políticas públicas e o Serviço Social. Serv. Soc. Soc. [online], n.105, p. 146-165, 2011. Disponível em: http://www.scielo.br/pdf/sssoc/n105/09.pdf. Acesso em: 16 ago. 2014.

MARMOT, M.; ATKINSON, T.; BELL, J. Fair Society, healthy lives: The Marmot Review. Strategic review of health inequalities in England Post, 2010.

MERHY, E. E.; FRANCO, T. B. Reestruturação produtiva em saúde. In: PEREIRA, I. B.; LIMA, J. C. F. (Org.). Dicionário da educação profissional em saúde. 2. ed. Rio de Janeiro: $\quad$ EPSJV; 2008. Disponível em: http://www.epsjv.fiocruz.br/dicionario/verbetes/reeprosau.html. Acesso em: 16 ago. 2014.

NOVAES, H. M. D. Da produção à avaliação de tecnologias dos sistemas de saúde: desafios do século XXI. Rev Saúde Pública, v. 40, p. 133-140, 2006. Número especial. Disponível em: http://www.scielo.br/pdf/rsp/v40nspe/30632.pdf. Acesso em: 16 ago. 2014.

OLIVEIRA, A. A. P. et al. Temas relevantes para a formação profissional em desenvolvimento infantil: um estudo de caso à luz da promoção da saúde. Revista Medicina (USP), v. 92, n. 2, p. 113-118, 2013. Disponível em: http://www.revistas.usp.br/revistadc/article/viewFile/79949/83884. Acesso em: 16 ago. 2014.

PATTON, M. Q. Developmental evaluation: applying complexity concepts to enhance innovation and use. New York: The Guilford Press, 2011. Disponível em: http://books.google.com.br/books?hl=pt-

BR\&lr=\&id=cd8yAAAAQBAJ \&oi=fnd $\& p g=P P 2 \& d q=$ parsons + social+innovation+evalua tion\&ots=R9doiySnGO\&sig=uwTVuwhmKzGxViCRMOBxOI4cO6g\#v=onepage \&q\&f=f alse. Acesso em: 16 ago. 2014.

RODRIGUES, I.; BARBIERI, J. Carlos. A emergência da tecnologia social: revisitando o movimento da tecnologia apropriada como estratégia de desenvolvimento sustentável. Rev. Adm. Pública [online], v. 42, n.6, p. 1069-1094, 2008. Disponível em: http://www.scielo.br/pdf/rap/v42n6/03.pdf. Acesso em: 16 ago. 2014.

SHONKOFF, J. P. et al. The lifelong effects of early childhood adversity and toxic stress. Pediatrics, n. 129, p. 232-246, 2012. Disponível em: http://pediatrics.aappublications.org/content/early/2011/12/21/peds.2011-

2663.full.pdf+html. Acesso em: 16 ago. 2014.

VERÍSSIMO, M. L. O. R.; CHIESA, A. M. A promoção do desenvolvimento infantil: instrumento para o acompanhamento dos cuidados familiares. In: FUJIMORI, E.; OHARA, C. V. S. (Org.). Enfermagem e a saúde da criança na atenção básica. Barueri: Manole, 2009. p. 328-53.

WORLD HEALTH ORGANIZATION. Transformative scale up of health professional education. Geneva: World Health Organization, 2011.

YIN, R. Estudo de caso: planejamento e métodos. 4.ed. Porto Alegre: Bookman, 2010.

YOUNG, M. E. (Org.). Do desenvolvimento da primeira infância ao desenvolvimento humano: investindo no futuro de nossas crianças. São Paulo: Fundação Maria Cecília Souto Vidigal, 2010. 